\title{
Article \\ Relative Symmetric Reductions under Multi-Choice Non-Transferable-Utility Situations
}

\author{
Yu-Hsien Liao
}

Citation: Liao, Y.-H. Relative Symmetric Reductions under Multi-Choice Non-TransferableUtility Situations. Mathematics 2022, 10, 682. https://doi.org/ $10.3390 /$ math10050682

Academic Editor: David Carfî

Received: 8 February 2022

Accepted: 20 February 2022

Published: 22 February 2022

Publisher's Note: MDPI stays neutral with regard to jurisdictional claims in published maps and institutional affiliations.

Copyright: (c) 2022 by the authors. Licensee MDPI, Basel, Switzerland. This article is an open access article distributed under the terms and conditions of the Creative Commons Attribution (CC BY) license (https:/ / creativecommons.org/licenses/by/ $4.0 /)$.
Department of Applied Mathematics, National Pingtung University, Pingtung City 900391, Taiwan; twincos@ms25.hinet.net or twincos@mail.nptu.edu.tw; Tel.: +886-958-631-010

\begin{abstract}
In many game-theoretical results, the reduction axiom and its converse have been regarded as important requirements under axiomatic processes for solutions. However, it is shown that the replicated core counters a specific (inferior) converse reduction axiom under multi-choice nontransferable-utility situations. Thus, two modified reductions and relative properties of the reduction axiom and its converse are proposed to characterize the replicated core in this article.The main methods and relative results are as follows. First, two different types of reductions are proposed by focusing on both participants and participation levels under relative symmetric reducing behavior. Further, relative reduction axioms and their converse are adopted to characterize the replicated core.
\end{abstract}

Keywords: non-transferable-utility situation; the replicated core; relative symmetric reducing behavior; reduction axiom

MSC: 91A; 91B

\section{Introduction}

The reduction axiom (reduced game property) and converse reduction axiom (converse reduced game property) are crucial properties of feasible solutions in the characteristic formulation of cooperative situations. The reduction axiom permits one to infer, from the attractiveness of a consequence for some issue, the attractiveness of its restraint to each subdivision of a group for the correlative reduction the subdivision confronts; as its name demonstrates, the converse reduction axiom empowers a converse functioning: to infer the attractiveness of a consequence for several issues from the attractiveness of its restraint to every subdivision for the correlative reduction this subdivision confronts. The converse reduction axiom might be regarded as an attribute of "decentralizability". Taking several situations, it should be picked for the situation involving the entire collection if an alternative is picked for each of its correlative reductions. The core is, probably, the most intuitional solution concept. There exist two distinct forms of reductions among the axiomatic results for the core in existing studies, the "max-reduction" (Davis and Maschler [1]) and the "complement-reduction" (Moulin [2]). For instance, Peleg [3,4], Serrano and Volij [5] characterized the core by means of the notion of the max-reductions. In addition, Tadenuma [6] characterized the core by applying complement-reductions.

A non-transferable-utility (NTU) situation could be regarded as a measuring of outcomes achievable by participants of every alliance through some joint procedures of decision (strategy). The participants confront the issue of picking an outcome that is feasible for the whole environment. On the other hand, a multi-choice NTU situation is a generalized analogue of a traditional NTU situation. Under a traditional NTU situation, each participant is either thoroughly involved or not involved radically under operation with some other participants, while under a multi-choice situation, every participant is permitted to adopt finite many distinct participation levels. As is known, solution concepts under multi-choice situations have been used in many fields, such as economics, environmental sciences, management sciences and even political sciences. Inspired by van den Nouweland et al. [7], 
Hwang and Li [8] introduced an extended core on multi-choice NTU situations. Later, Hwang and Liao [9] proposed the replicated core by extending the core concept of Hwang and Liao [10] to multi-choice NTU situations. Further, Hwang and Liao [9] defined a multichoice analogue of Peleg's [3] reduction to characterize the replicated core. Hwang and Liao [9] also provided an extended complement-reduction on multi-choice NTU situations. However, Hwang and Liao [9] presented that the replicated core is "not" the unique solution matching individual rationality, non-emptiness and complement-reduction axiom under the domain of multi-choice NTU balanced situations. The cause is that the replicated core counters the (inferior) converse complement-reduction axiom.

As mentioned above, one motivation could be considered worthy:

- Whether different types of reductions, related reduction axioms and their converse could be proposed to characterize the replicated core.

To resolve this motivation, one would build on the results of Hwang and Liao [9]. Relative results are as follows.

1. Different from the reductions due to Hwang and Liao [9], two different types of the reductions are generated in Section 3 by focusing on both participants and participation levels under relative symmetric reducing behavior. These reductions are introduced to multi-choice NTU situations and relative reduction axioms and their converse are presented.

2. To present the applied expedience and the mathematical correctness simultaneously, the replicated core is characterized in Section 4 by means of relative reduction axioms and their converse. Further, the findings of this article are compared with existing results.

\section{Preliminaries}

Let $U P$ be the universe of participants. Assume that every participant $t$ has $h_{t} \in \mathbb{P}$ participation levels at which it can operate. Let $h^{U P} \in \mathbb{P}^{U P}$ be the vector that presents the amount of participation levels for every participant. Let $P \subseteq U P$ be a collection of participants. Denote $h_{P}^{U P} \in \mathbb{R}^{P}$ as the restriction of $h^{U P}$ to $P$. For $t \in U P$, we set $H_{t}=\left\{0,1, \cdots, h_{t}\right\}$ as the participation level space of participant $t$, where 0 means not participating and $H_{t}^{+}=H_{t} \backslash\{0\}$. For $P \subseteq U P, P \neq \varnothing$, let $H^{P}=\prod_{t \in P} H_{t}$ be the product collection of the participation level spaces for participants $P$. Given $\alpha \in \mathbb{R}^{P}$, we define that $E(\alpha)=\left\{t \in P \mid \alpha_{t}>0\right\}$ and $\alpha_{T} \in \mathbb{R}^{T}$ as the restriction of $\alpha$ to coalition $T$.

Let $\alpha, \beta \in \mathbb{R}^{P} . \alpha \geq \beta$ if $\alpha_{i} \geq \beta_{i}$ for every $i \in P ; \alpha>\beta$ if $\alpha \geq \beta$ and $\alpha \neq \beta ; \alpha \gg \beta$ if $\alpha_{i}>\beta_{i}$ for every $i \in P$. Denote that $\mathbb{R}_{+}^{P}=\left\{\alpha \in \mathbb{R}^{P} \mid \alpha \geq 0_{P}\right\}$. Let $K \subseteq \mathbb{R}^{P}$. K is comprehensive if $\alpha \in K$ and $\alpha \geq \beta$ imply $\beta \in K$. Denote the comprehensive hull of $K$ as $\operatorname{com} K$, the boundary of $K$ as $\partial K$ and the interior of $K$ as int $K$. If $\alpha \in \mathbb{R}^{P}$, then $\alpha+K=\{\alpha+k \mid k \in K\}$.

Definition 1. A multi-choice NTU situation is denoted by $(P, M)$ (as $h^{U P} \in \mathbb{P}^{U P}$ is fixed in the article, one could write $(P, M)$ rather than $\left(P, h_{P}^{U P}, M\right)$. Thus, $h_{P}^{U P}$ will be denoted by $h$ so no confusion can occur), where $P \neq \varnothing$ is a finite collection of participants and $M$ is a measuring function that allots to every $\alpha=\left(\alpha_{i}\right)_{i \in P} \in H^{P} \backslash\left\{0_{P}\right\}$ a subset $M(\alpha)$ of $\mathbb{R}^{E(\alpha)}$ matching:

1. $M(\alpha)$ is closed, non-empty and comprehensive.

2. $M(\alpha) \cap\left(\lambda+\mathbb{R}_{+}^{E(\alpha)}\right)$ is bounded for all $\lambda \in \mathbb{R}^{E(\alpha)}$.

Denote the class of total multi-choice NTU situations as $\overline{N G}$. Taking $T \subseteq P$, let $|T|$ be the amount of elements in $T$, and let $e^{T}(P)=\left(e_{i}^{T}(P)\right)_{i \in P} \in \mathbb{R}^{P}$ with

$$
e_{i}^{T}(P)= \begin{cases}1 & \text { if } i \in T \\ 0 & \text { otherwise }\end{cases}
$$

Here, $e^{T}(P)$ is denoted as $e^{T}$ so no confusion can arise. 
Let $(P, M) \in \overline{N G}$. A payoff vector of $(P, M)$ is a vector $\left(\lambda_{i}\right)_{i \in P} \in \mathbb{R}^{P}$, where $\lambda_{i}$ shows the per unit payoff that participant $i$ obtains for every $i \in P$, hence $h_{i} \lambda_{i}$ is the global payoff that participant $i$ obtains at $(P, M)$. Then, a payoff vector $\lambda$ is

- efficient if $\left(h_{t} \lambda_{t}\right)_{t \in P} \in \partial M(h)$.

- individually expedient if for all $t \in P$ and for all $k_{t} \in H_{t}^{+}, k_{t} \lambda_{i} \notin \operatorname{int} M\left(k_{t} e^{\{t\}}\right)$.

In addition, $\lambda$ is an imputation of $(P, M)$ if it is efficient and expedient. We denote collection of feasible payoff vectors of $(P, M)$ as

$$
X^{*}(P, M)=\left\{\lambda \in \mathbb{R}^{P} \mid\left(h_{i} \lambda_{i}\right)_{i \in P} \in M(h)\right\},
$$

whereas

$$
X(P, M)=\left\{\lambda \in \mathbb{R}^{P} \mid \lambda \text { is EFF }\right\}
$$

is the collection of preimputations of $(P, M)$ and the collection of imputations of $(P, M)$ is

$$
I(P, M)=\left\{\lambda \in \mathbb{R}^{P} \mid \lambda \text { is an imputation of }(P, M)\right\} .
$$

A solution on $\overline{N G}$ is a mapping $\tau$ which associates with every $(P, M) \in \overline{N G}$ a subset $\tau(P, M)$ of $X^{*}(P, M)$. Hwang and Liao [9] proposed a solution concept as follows.

Definition 2. (Hwang and Liao [9]). Let $(P, M) \in \overline{N G}$. The replicated core $R C(P, M)$ of $(P, M) \in \overline{N G}$ is defined by

$$
R C(P, M)=\left\{\lambda \in X(P, M) \mid\left(\alpha_{i} \lambda_{i}\right)_{i \in E(\alpha)} \notin \operatorname{int} M(\alpha) \text { for all } \alpha \in M^{P} \backslash\left\{0_{P}\right\}\right\} .
$$

\section{Axioms and Reductions}

Let $\tau$ be a solution on $\overline{N G}$. $\tau$ matches non-emptiness (NES) if for all $(P, M) \in \overline{N G}$, $\tau(P, M) \neq \varnothing . \tau$ matches efficiency (EIY) if for all $(P, M) \in \overline{N G}, \tau(P, M) \subseteq X(P, M)$. $\tau$ matches individual expediency (IEY) if for all $(P, M) \in \overline{N G}, \tau(P, M) \subseteq I(P, M)$. $\tau$ matches one-person expediency (OPEY) if for all $(P, M) \in \overline{N G}$ with $|P|=1, \tau(P, M)=$ $I(P, M)$.

Inspired by Peleg [4] and Moulin [2], Hwang and Liao [9] introduced two extended reductions by only focusing on participants as follows. Given $(P, M) \in \overline{N G}, T \subseteq P$ with $T \neq \varnothing$ and a payoff vector $\lambda$, the DM-reduction is the situation $\left(T, M_{T, \lambda}\right)$ defined by for all $\alpha \in H^{T}$,

$$
\begin{array}{ll}
M_{T, \lambda}(\alpha)=\left\{\omega \in \mathbb{R}^{T} \mid\left(\omega, \lambda_{P \backslash T}\right) \in M(h)\right\} & , \text { if } \alpha=h_{T}, \\
M_{T, \lambda}(\alpha)=\bigcup_{\beta \in M^{P \backslash T}}\left\{\omega \in \mathbb{R}^{E(\alpha)} \mid\left(\omega,\left(\beta_{i} \lambda_{i}\right)_{i \in E(\beta)}\right) \in M(\alpha, \beta)\right\} & , \text { otherwise. }
\end{array}
$$

The M-reduction is the situation $\left(T, M^{T, \lambda}\right)$ defined by for all $\alpha \in H^{T}$,

$$
M^{T, \lambda}(\alpha)=\left\{\omega \in \mathbb{R}^{E(\alpha)} \mid\left(\omega,\left(h_{i} \lambda_{i}\right)_{i \in P \backslash T}\right) \in M\left(\alpha, h_{P \backslash T}\right)\right\} .
$$

The reduction axiom, originally defined by Harsanyi [11] under the name of bilateral equilibrium, claims that the projection of $\lambda$ to $P$ should be stipulated by $\tau$ for the reduced condition with regard to $P$ and $\lambda$ if $\lambda$ is stipulated by $\tau$ for a situation $(P, M)$. Hence, the projection of $\lambda$ to $P$ should be reduction axiomatic with the expectations of the components of $P$ as reflected by its reduced condition.

- DM-reduction axiom (DMRDA): If $(P, M) \in \overline{N G}, T \subseteq P, T \neq \varnothing$, and $\lambda \in \tau(P, M)$, then $\left(T, M_{T, \lambda}\right) \in \overline{N G}$ and $\lambda_{T} \in \tau\left(T, M_{T, \lambda}\right)$.

The converse reduction axiom claims that $\lambda$ itself should be appointed for entire situation if the projection of an efficient payoff vector $\lambda$ to each proper $P$ is reduction axiomatic with the expectations of the components of $P$ as reflected by its reduced condition. 
- Converse DM-reduction axiom (CDMRDA): If $(P, M) \in \overline{N G}$ with $|P| \geq 2, \lambda \in$ $X(P, M)$, and for all $T \subset P, 0<|T|<|P|,\left(T, M_{T, \lambda}\right) \in \overline{N G}$ and $\lambda_{T} \in \tau\left(T, M_{T, \lambda}\right)$, then $\lambda \in \tau(P, M)$.

The following axiom is a weakening of the converse reduction axiom, since it claims that $\lambda$ is individually expedient as well.

- Inferior converse DM-reduction axiom (ICDMRDA): If $(P, M) \in \overline{N G}$ with $|P| \geq 2$, $\lambda \in I(P, M)$, and for all $T \subset P, 0<|T|<|P|,\left(T, M_{T, \lambda}\right) \in \overline{N G}$ and $\lambda_{T} \in \tau\left(T, M_{T, \lambda}\right)$, then $\lambda \in \tau(P, M)$.

For M-reduction instead of DM-reduction, Hwang and Liao [9] proposed the M-reduction axiom (MRDA), converse M-reduction axiom (CMRDA) and inferior converse M-reduction axiom (ICMRDA).

Remark 1. Hwang and Liao [9] characterized the replicated core by means of OPEY, IEY, DMRDA and ICDMRDA. Hwang and Liao [9] also showed that the replicated core matches MRDA and violates CMRDA (ICMRDA).

Subsequently, two extended reductions are proposed by focusing on participants and participation levels, respectively.

Definition 3. Given $(P, M) \in \overline{N G}, T \subseteq P$ with $T \neq \varnothing$, a payoff vector $\lambda$ and $\gamma \in H_{+}^{P \backslash T}$. The relative symmetric DM-reduction, $\left(T, M_{\lambda, T, \gamma}\right)$, is defined by for all $\alpha \in H^{T} \backslash\left\{0_{T}\right\}$,

$$
\begin{array}{ll}
M_{\lambda, T, \gamma}(\alpha)=\left\{\omega \in \mathbb{R}^{T} \mid\left(\omega, \lambda_{P \backslash T}\right) \in M(h)\right\} & \text {, if } \alpha=h_{T}, \\
M_{\lambda, T, \gamma}(\alpha)=\bigcup_{Q \subseteq P \backslash T}\left\{\omega \in \mathbb{R}^{E(\alpha)} \mid\left(\omega,\left(\gamma_{i} \lambda_{i}\right)_{i \in Q}\right) \in M\left(\alpha, \gamma_{Q}, 0_{(P \backslash T) \backslash Q}\right)\right\} & \text {, otherwise. }
\end{array}
$$

The relative symmetric M-reduction, $\left(T, M^{\lambda, T, \gamma}\right)$, is defined by for all $\alpha \in H^{T} \backslash\left\{0_{T}\right\}$,

$$
\begin{array}{ll}
M^{\lambda, T, \gamma}(\alpha)=\left\{\omega \in \mathbb{R}^{T} \mid\left(\omega, \lambda_{P \backslash T}\right) \in M(h)\right\} & , \text { if } \alpha=h_{T}, \\
M^{\lambda, T, \gamma}(\alpha)=\left\{\omega \in \mathbb{R}^{E(\alpha)} \mid\left(\omega,\left(\gamma_{i} \lambda_{i}\right)_{i \in P \backslash T}\right) \in M(\alpha, \gamma)\right\} & , \text { otherwise. }
\end{array}
$$

Note that the participation level $\gamma \in H_{+}^{P \backslash T}$ indicates that the participants of $P \backslash T$ would symmetrically proceed to collaborate with the participants of $T$ if renegotiating the payoff allocation amid $T$. Total participants in $P \backslash T$ adopt non-zero participation levels based on the participation vector $\gamma$ to collaborate symmetrically.

For relative symmetric DM-reduction instead of DM-reduction, one could introduce the relative symmetric DM-reduction axiom (RSDMRDA), converse relative symmetric DM-reduction axiom (CRSDMRDA) and inferior converse relative symmetric DMreduction axiom (ICRSDMRDA). Additionally, for relative symmetric M-reduction instead of DM-reduction, one could introduce the relative symmetric M-reduction axiom (RSMRDA), converse relative symmetric M-reduction axiom (CRSMRDA) and inferior converse relative symmetric M-reduction axiom (ICRSMRDA).

\section{Main Results}

In this section, we adopt OPEY, RSDMRDA (RSMRDA) and CRSDMRDA (CRSMRDA) to characterize the replicated core.

Lemma 1. Let $(P, M) \in \overline{N G}, \lambda \in M(h), T \subseteq P$ with $T \neq \varnothing$, and $\gamma \in H_{+}^{P \backslash T}$. Then, $\left(T, M_{\lambda, T, \gamma}\right) \in \overline{N G}$ and $\left(T, H^{\lambda, T, \gamma}\right) \in \overline{N G}$.

Proof of Lemma 1. The verification of this lemma is straightforward. Thus, it is omitted.

Lemma 2. Let $(P, M) \in \overline{N G}, \lambda \in M(h), T \subseteq P$ with $T \neq \varnothing$, and $\gamma \in H_{+}^{P \backslash T}$. Then, 
1. $\lambda \in X(P, M)$ if and only if $\lambda_{T} \in X\left(T, M_{\lambda, T, \gamma}\right)$.

2. $\lambda \in X(P, M)$ if and only if $\lambda_{T} \in X\left(T, H^{\lambda, T, \gamma}\right)$.

Proof of Lemma 2. To prove $1, \lambda_{T} \in M_{\lambda, T, \gamma}\left(h_{T}\right)$ absolutely. If $\lambda_{T} \notin X\left(T, M_{\lambda, T, \gamma}\right)$, then there exists $\omega_{T} \in M_{\lambda, T, \gamma}\left(h_{T}\right)$ such that $\omega_{T}>\lambda_{T}$. Hence, $\left(\omega_{T}, \lambda_{P \backslash T}\right) \in M(h)$ and $\left(\omega_{T}, \lambda_{P \backslash T}\right)>\lambda$. Therefore, $\lambda \notin X(P, M)$. Similarly, if $\lambda \notin X(P, M)$, then there exists $\omega \in M(h)$ such that $\omega \gg \lambda$. So, $\left(\omega_{T}, \lambda_{P \backslash T}\right) \in M(h)$. Thus, $\omega_{T} \in M_{\lambda, T, \gamma}\left(h_{T}\right)$ and $\omega_{T} \gg \lambda_{T}$. Hence, $\lambda_{T} \notin X\left(T, M_{\lambda, T, \gamma}\right)$. The proof of 2 is similar.

\section{Lemma 3.}

1. The replicated core matches $R S D M R D A$.

2. The replicated core matches $R S M R D A$.

Proof of Lemma 3. Let $(P, M) \in \overline{N G}$. Let $\lambda \in R C(P, M), T \subseteq P$ with $T \neq \varnothing$, and $\gamma \in$ $H_{+}^{P \backslash T}$. To prove 1, by Lemma $1,\left(T, M_{\lambda, T, \gamma}\right) \Gamma$. We show that $\left(T, M_{\lambda, T, \gamma}\right) \in \overline{N G}$ and $\lambda_{T} \in$ $R C\left(T, M_{\lambda, T, \gamma}\right)$. Since $\lambda \in R C(P, M), \lambda \in X(P, M)$. Thus, $\lambda_{T} \in X\left(T, M_{\lambda, T, \gamma}\right)$ by Lemma 2 . It remains to be shown that for all $\alpha \in H^{T} \backslash\left\{0_{T}, h_{T}\right\}$,

$$
\left(\alpha_{i} \lambda_{i}\right)_{i \in E(\alpha)} \notin \operatorname{int} M_{\lambda, T, \gamma}(\alpha) .
$$

Assume, on the contrary, that there exists $\alpha \in H^{T} \backslash\left\{0_{T}, h_{T}\right\}$ such that $\left(\alpha_{i} \lambda_{i}\right)_{i \in E(\alpha)} \in$ $\operatorname{int} M_{\lambda, T, \gamma}(\alpha)$, that is,

$$
\left(\alpha_{i} \lambda_{i}\right)_{i \in E(\alpha)} \in \operatorname{int}\left[\bigcup_{Q \subseteq P \backslash T}\left\{\omega \in \mathbb{R}^{E(\alpha)} \mid\left(\omega,\left(\gamma_{i} \lambda_{i}\right)_{i \in Q}\right) \in M\left(\alpha, \gamma_{Q}, 0_{(P \backslash T) \backslash Q}\right)\right\}\right] .
$$

Then, there exists $Q \subseteq P \backslash T$ such that

$$
\left(\left(\alpha_{i} \lambda_{i}\right)_{i \in E(\alpha)},\left(\gamma_{i} \lambda_{i}\right)_{i \in Q}\right) \in \operatorname{int} M\left(\alpha, \gamma_{Q}, 0_{(P \backslash T) \backslash Q}\right) .
$$

Let $\bar{\alpha}=\left(\alpha, \gamma_{Q}, 0_{(P \backslash T) \backslash Q}\right) \in H^{P} \backslash\left\{0_{P}\right\}$. Thus, $\left(\bar{\alpha}_{i} \lambda_{i}\right)_{i \in E(\bar{\alpha})} \in$ int $M(\bar{\alpha})$. This contradicts that $\lambda \in R C(P, M)$. The proof of 2 is similar.

\section{Lemma 4.}

1. The replicated core matches CRSDMRDA.

2. The replicated core matches CRSMRDA.

Proof of Lemma 4. Let $(P, M) \in \overline{N G}$ with $|P| \geq 3$ and let $\lambda \in M(h)$. To prove 1 , suppose that for all $T \subset P$ with $|T|=2$ and for all $\gamma \in H_{+}^{P \backslash T},\left(T, M_{\lambda, T, \gamma}\right) \in \overline{N G}$ and $\lambda_{T} \in$ $R C\left(T, M_{\lambda, T, \gamma}\right)$. We show that $\lambda \in R C(P, M)$. Since $\lambda_{T} \in R C\left(T, M_{\lambda, T, \gamma}\right), \lambda_{T}$ is EIY in $\left(T, M_{\lambda, T, \gamma}\right)$. Thus, $\lambda$ is EIY in $(P, M)$ by Lemma 2 . It remains to be shown that for all $\alpha \in H^{P} \backslash\left\{0_{P}, m\right\},\left(\alpha_{i} \lambda_{i}\right)_{i \in E(\alpha)} \notin$ int $M(\alpha)$. Assume, on the contrary, that there exists $\alpha \in H^{P} \backslash\left\{0_{P}, m\right\}$ such that $\left(\alpha_{i} \lambda_{i}\right)_{i \in E(\alpha)} \in \operatorname{int} M(\alpha)$. Two conditions can be distinguished: Condition 1: $E(\alpha)=P$ :

Choose $k \in E(\alpha)$ with $\alpha_{k} \neq 1$ (this could be completed since $\alpha \neq h$ ). Let $j \in P, j \neq k$, and let $T=\{k, j\}, \gamma=\alpha_{P \backslash T}$. Since $\left(\alpha_{i} \lambda_{i}\right)_{i \in E(\alpha)} \in \operatorname{int} M(\alpha)$ and

$$
M_{\lambda, T, \gamma}\left(\alpha_{k}, \alpha_{j}\right)=\bigcup_{Q \subseteq P \backslash T}\left\{\omega \in \mathbb{R}^{\{k, j\}} \mid\left(\omega,\left(\gamma_{i} \lambda_{i}\right)_{i \in Q}\right) \in M\left(\alpha_{\{k, j\}}, \gamma_{Q}, 0_{(P \backslash T) \backslash Q}\right)\right\},
$$

by adopting $Q=P \backslash T$, we have that $\left(\alpha_{k} \lambda_{k}, \alpha_{j} \lambda_{j}\right) \in \operatorname{int} M_{\lambda, T, \gamma}\left(\alpha_{k}, \alpha_{j}\right)$.

Condition 2: $E(\alpha) \neq P$ :

Choose $k \in E(\alpha)$. Let $j \notin E(\alpha)$, and let $T=\{k, j\}$. For convenience, let $T=P \backslash(E(\alpha) \cup\{j\})$. 
We adopt $\gamma=\left(\alpha_{E(\alpha) \backslash\{k\}}, h_{T}\right)$. Since $\alpha_{j}=0$, by the same processes as Condition 1 except adopting $Q=E(\alpha) \backslash\{k\}$, we derive that $\alpha_{k} \lambda_{k} \in \operatorname{int} M_{\lambda, T, \gamma}\left(\alpha_{k}, 0\right)$.

By Conditions 1 and $2, \lambda_{T} \notin R C\left(T, M_{\lambda, T, \gamma}\right)$, the desired contradiction has been generated. The proof of 2 is similar.

\section{Lemma 5.}

1. A solution $\tau$ on $\overline{N G}$ matches EIY if it matches OPEY and RSDMRDA.

2. A solution $\tau$ on $\overline{N G}$ matches EIY if it matches OPEY and RSMRDA.

Proof of Lemma 5. The proof can easily be deduced from Lemma 5.4 in Peleg [3].

\section{Theorem 1.}

1. A solution $\sigma$ on $\overline{N G}$ matches OPEY, RSDMRDA and CRSDMRDA if and only if for all $(P, M) \in \overline{N G}, \sigma(P, M)=R C(P, M)$.

2. A solution $\sigma$ on $\overline{N G}$ matches OPEY, RSMRDA and CRSMRDA if and only if for all $(P, M) \in \overline{N G}, \sigma(P, M)=R C(P, M)$.

Proof of Theorem 1. By Lemmas 3 and 4, the replicated core matches RSDMRDA, RSMRDA, CRSDMRDA and CRSMRDA. Hwang and Liao [9] showed that it matches OPEY.

To present the uniqueness of 1 , assume that a solution $\sigma$ matches OPEY, RSDMRDA and CRSDMRDA. By Lemma $5, \sigma$ matches EIY. Let $(P, M) \in \overline{N G}$. The proof proceeds by induction on $|P| . \sigma(P, M)=I(P, M)=R C(P, M)$ by OPEY of $\sigma$ if $|P|=1$. Assume that $\sigma(P, M)=R C(P, M)$ if $|P|<k, k \geq 2$. The condition $|P|=k$ :

First, we prove that $\sigma(P, M) \subseteq R C(P, M)$. Let $\lambda \in \sigma(P, M)$. By RSDMRDA of $\sigma$, for all $T \subset P$ with $0<|T|<|P|, \lambda_{T} \in \sigma\left(T, M_{\lambda, T, \gamma}\right)$. By the induction hypothesis, $\lambda_{T} \in$ $\sigma\left(T, M_{\lambda, T, \gamma}\right)=R C\left(T, M_{\lambda, T, \gamma}\right)$. Since $\lambda \in X(P, M)$, by CRSDMRDA of $R C, \lambda \in R C(P, M)$. The opposite inclusion could be completed analogously by exchanging the parts of $\sigma$ and $C$. Thus, $\sigma(P, M)=R C(P, M)$. The proof of the uniqueness of 2 is similar.

The following examples present that each of the axioms taken in Theorem 1 is logically independent of the others. Clearly, $|U P| \geq 2$ is needed.

Example 1. Let $\sigma(P, M)=\varnothing$ for all $(P, M) \in \overline{N G}$. Then, $\sigma$ matches RSDMRDA, RSMRDA, CRSDMRDA and CRSMRDA, but it counters OPEY.

Example 2. Let $\sigma(P, M)=I(P, M)$ for all $(P, M) \in \overline{N G}$. Then, $\sigma$ matches OPEY, CRSDMRDA and CRSMRDA, but it counters RSDMRDA and RSMRDA.

Example 3. Define a solution $\sigma$ on $\overline{N G}$ by

$$
\sigma(P, M)= \begin{cases}I(P, M) & , \text { if }|P|=1 \\ \varnothing & , \text { otherwise. }\end{cases}
$$

Then, $\sigma$ matches OPEY, RSDMRDA and RSMRDA, but it counters CRSDMRDA and CRSMRDA.

\section{Discussion and Conclusions}

1. In real-world situations, participants and their participation behavior have been regarded as essential factors under operational processes of interactive situations. In the frameworks of traditional situations and multi-choice NTU situations, however, reductions and relative properties of the reduction axiom and its converse have been defined by only focusing on participants. Thus, different from existing traditional situations and multi-choice NTU situations, the main results of Hwang and Liao [9] are extended under multi-choice NTU situations. The main results of this article are compared with relative existing results in the frameworks of traditional situations and multi-choice NTU situations as follows. 
- $\quad$ Under multi-choice NTU situations, the reductions due to Hwang and Liao [9] are defined by only focusing on participants. In this article, two reductions are introduced by only focusing on participants and participation levels under relative symmetric reducing behavior. This revising notion does not appear in Hwang and Liao [9] and the frameworks of traditional situations and multichoice NTU situations.

- $\quad$ Based on the extended M-reduction due to Hwang and Liao [9], the replicated core could not be characterized by the relative (inferior) converse reduction axiom. Based on the two extended reductions of this article, however, the replicated core could be characterized by relative (inferior) converse reduction axioms. More precisely, this article shows that the replicated core is the only solution matching one-person expediency, relative symmetric DM-reduction axiom (relative symmetric M-reduction axiom) and converse relative symmetric DM-reduction axiom (converse relative symmetric M-reduction axiom). These axiomatic results do not appear in Hwang and Liao [9] and the frameworks of traditional situations and multi-choice NTU situations.

- $\quad$ The axiomatic techniques of Hwang and Liao [9] and this article are exactly corresponding to the relative techniques of Serrano and Volij [5].

2. The reduction axiom and (inferior) converse reduction axiom of a solution are indispensable under axiomatic techniques of existing studies of core concepts. However, some extended core concepts always violate the reduction axiom or (inferior) converse reduction axiom under multi-choice NTU situations. In future studies, one could attempt to characterize these core concepts by putting down the reduction axiom or (inferior) converse reduction axiom.

Funding: This research received no external funding.

Institutional Review Board Statement: Not applicable.

Informed Consent Statement: Not applicable.

Data Availability Statement: Not applicable.

Conflicts of Interest: The author declares no conflict of interest.

\section{References}

1. Davis, M.; Maschler, M. The kernel of a cooperative game. Nav. Res. Logist. Q. 1965, 12, 223-259. [CrossRef]

2. Moulin, H. The separability axiom and equal-sharing methods. J. Econ. Theory 1985, 36, 120-148. [CrossRef]

3. Peleg, B. An axiomatization of the core of cooperative games without side payments. J. Math. Econ. 1985, 14, 203-214. [CrossRef]

4. Peleg, B. On the reduced game property and its converse. Int. J. Game Theory 1986, 15, 187-200. [CrossRef]

5. Serrano, R.; Volij, O. Axiomatizations of neoclassical concepts for economies. J. Math. Econ. 1998, 30, 87-108. [CrossRef]

6. Tadenuma, K. Reduced games, reduction axiom, and the core. Int. J. Game Theory 1992, 20, 325-334. [CrossRef]

7. van den, N.A.; Potters, J.; Tijs, S.; Zarzuelo, J. Core and relative solution concepts for multi-choice games. ZOR-Math. Methods Oper. Res. 1995, 41, 289-311.

8. Hwang, Y.A.; Li, W.H. The core of multi-choice NTU games. Math. Methods Oper. Res. 2005, 61, 33-40. [CrossRef]

9. Hwang, Y.A.; Liao, Y.H. A solution concept and its axiomatic results under non-transferable-utility and multi-choice situations. Mathematics 2020, 8, 1559. [CrossRef]

10. Hwang, Y.A.; Liao, Y.H. The unit-level-core for multi-choice games: The replicated core for TU games. J. Glob. Optim. 2010, 47, 161-171. [CrossRef]

11. Harsanyi, J.C. A bargaining model for the cooperative n-person game. In Contributions to the Theory of Games IV (Annals of Mathematics Studies); Tucker, A.W., Luce, R.D., Eds.; Princeton University Press: Princeton, NJ, USA, 1959; Volume 40, pp. 325-355. 